\title{
The use of peri neural catheters for post operative analgesia in lower limb amputation.
}

\author{
Frimley Health \\ NHS Foundation Trust \\ SN Phillips, R Hawkins, M Narayanan, H Say, J Stone, P Joshi. \\ Frimley Park Hospital UK
}

\section{BACKGROUND}

We have recently updated our guidelines for the management of post-operative analgesia for patients undergoing lower limb amputations. Pre-operative anti-neuropathic agents should be started, patients undergoing below knee amputations should receive sciatic nerve catheters and those undergoing through or above knee should have both sciatic and femoral nerve catheters placed.

We audited compliance with the new guidance, analgesia used, pain scores and patient satisfaction.

\section{METHODS}

Data was prospectively collected over a 5 -month period on:

- Type of analgesia,

- worst dynamic pains cores $(0=$ no pain, $3=$ severe pain $)$,

- analgesia used

- compliance with the Trust's guidelines

- patient satisfaction ( $0=$ very dissatisfied, $5=$ very satisfied $)$ for 4 days post operatively.

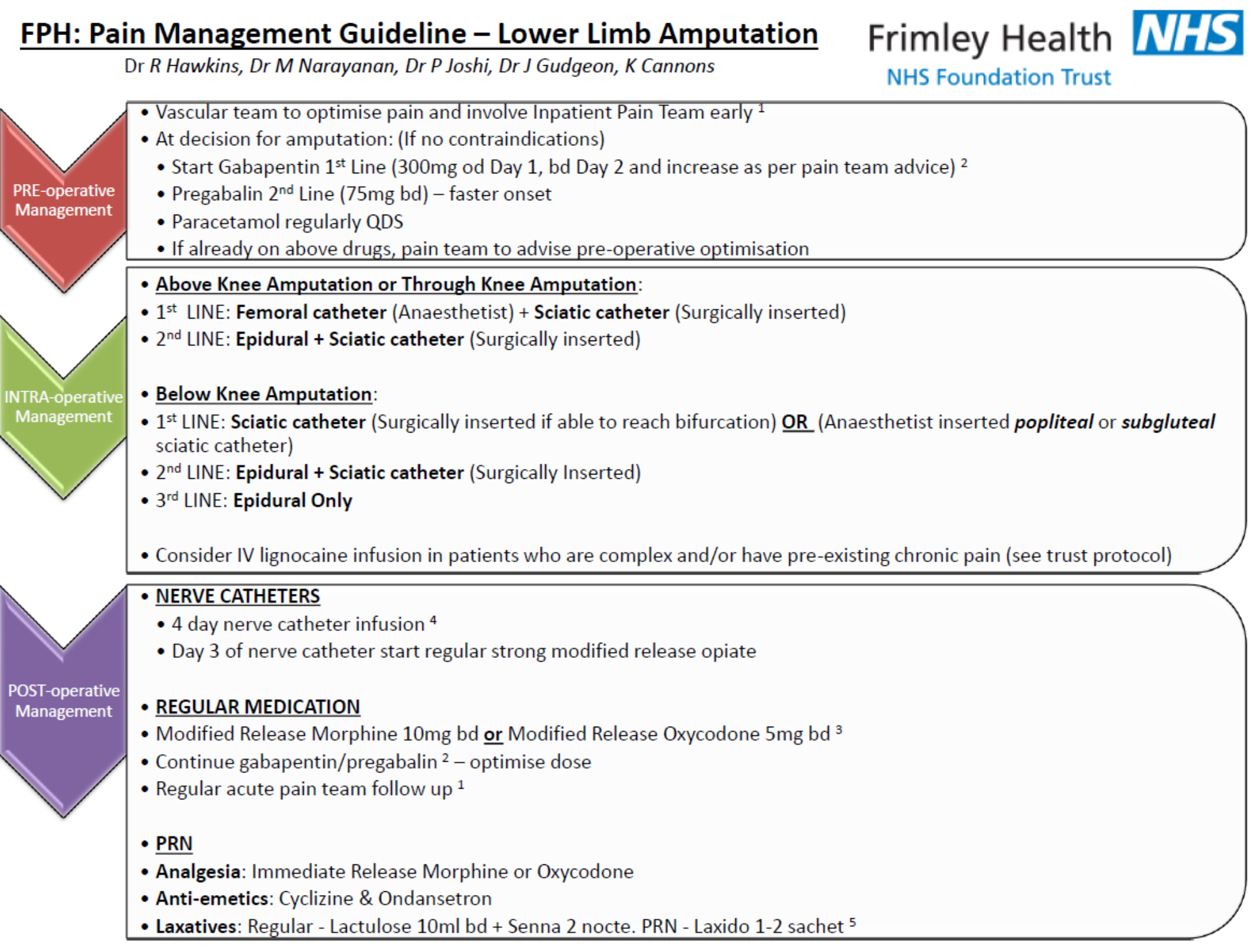

\section{RESULTS}

32 patients underwent lower limb amputations (20 below knee, 8 above knee, 3 through knee and 2 bilateral amputations).

$19 / 32$ patients received anti-neuropathic agents prior to surgery, this only increased to $20 / 32$ post op.

All patients received paracetamol, but only 2 patients received strong opioids. 1 patient had an NSAID and received 13 weak opioids, theses are not within the guidance.

The 2 patients undergoing bilateral amputations received epidurals.

8 patients did not have peri-neural catheters (PNC) as per the hospital guidelines.
The patients who received perineural catheters as per guidelines had lower pain scores (1.6 Vs 2.8) and fewer episodes of consecutively high pain scores compared to those management did not comply with guidance.

Patent satisfaction was similar in both groups.

Worst dynamic pain score Vs. day of assessment

40

30

20

10

0

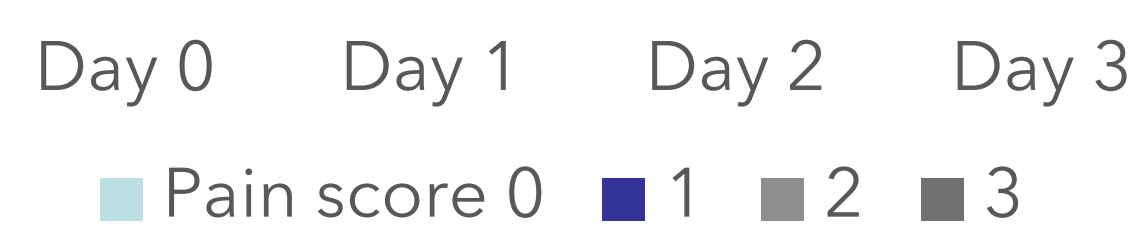

Average worst dynamic pain scores

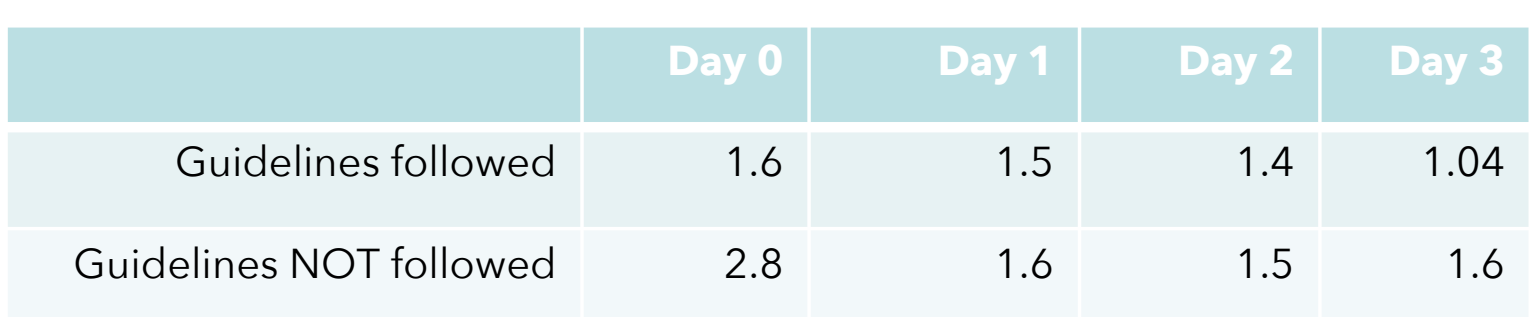

Number of patients who had consecutive pain scores of 2 or more

\begin{tabular}{|r|r|r|r|r|}
\hline $\begin{array}{r}\text { Guidelines followed } \\
\text { Guidelines NOT } \\
\text { followed }\end{array}$ & $6 / 20$ & $4 / 20$ & $2 / 20$ & $1 / 20$ \\
\hline
\end{tabular}

\section{CONCLUSIONS}

Lower limb amputations are painful, these patients require regular initial input from the pain team to help modify analgesia

We are good at prescribing paracetamol and avoiding NSAIDs, but need to improve the use of anti-neuropathic and strong opioids.

Compliance with guidelines provides better analgesia with lower pains scores.

We need to increase awareness of the guidelines and will provide training sessions on insertion of PNC for vascular anaesthetists to increase compliance and provide better analgesia in this group of patients. 